\title{
THE PREVALENCE OF CARDIOVASCULAR DISEASE RISK FACTORS IN NORMOTENSIVE, PRE-HYPERTENSIVE AND HYPERTENSIVE SOUTH AFRICAN COLLIERY EXECUTIVES
}

\author{
JEANNE GRACE and STUART SEMPLE \\ University of Zululand, KwaDlangezwa, Kwazulu Natal, South Africa \\ Faculty of Science and Agriculture, Department of Biokinetics and Sport Science
}

\begin{abstract}
Objective: The aim of this study was to establish the prevalence of cardiovascular disease (CVD) risk factors amongst South African colliery executives presenting normal blood pressure (normotensive), pre-hypertension and hypertension. Materials and Methods: Selected CVD risk factors of a non-randomized, available population of 143 Caucasian male executives from five South African collieries situated in Mpumalanga and Gauteng Provinces were recorded. Results: Executives with pre-hypertension and hypertension exhibited a higher prevalence of CVD risk factors, compared to the persons with normal blood pressure levels. The percentage of executives with CVD risk factors, with the exception of BMI, was greater amongst those with pre-hypertension than those with hypertension. Conclusion: The current study showed that a workplace CVD risk screening process was effective in identifying the relatively high prevalence of CVD risk factors amongst SA colliery executives. In addition, out of all the studied risk factors, undesirable body composition (BMI, WHR and fat \%) exhibited the highest prevalence amongst pre-hypertensive and hypertensive SA colliery executives.
\end{abstract}

Key words:

Executives, Blood pressure, Hypertension, Pre-hypertension, Cardiovascular disease

\section{INTRODUCTION}

Several epidemiologic studies have demonstrated that systolic and diastolic blood pressures have a "strong", continuous, graded and etiologically significant positive association with cardiovascular-disease outcomes [1-7]. Elevated blood pressure (BP) is a relatively common form of cardiovascular disease. It is associated with an increased risk of morbidity and mortality, and represents the single greatest preventable cause of deaths in humans [8,9]. Mortality data in South Africa show that nearly $25 \%$ of deaths may be related to cardiovascular disease (CVD) [10]. This trend is consistent in both men and women, in young, middle-aged, and older subjects and among different racial and ethnic groups [1-7]. The awareness, treatment, and control of high blood pressure have increased, but still remain insufficient [7,11-13]. Approximately six million South Africans aged 15 years and older suffer from hypertension, millions of whom were not diagnosed and even more inadequately treated. Out of these six million, only $26 \%$ of men and $51 \%$ of women were aware that they had hypertension [14]. In addition to this, it has been suggested that the prevalence of CVD and hypertension is increasing rapidly in sub-Saharan Africa [7,12].

Received: November 10, 2011. Accepted: April 27, 2012.

Address reprint request to J. Grace, PO Box 101805, Meerensee, Richards Bay, South Africa 3901 (e-mail: jgrace@pan.uzulu.ac.za). 
The Seventh Report of the National Committee on Prevention, Detection, Evaluation and Treatment of High Blood Pressure as well as the American College of Sports Medicine (ACSM) define persons with above-optimal levels but not clinical hypertension as having "prehypertension" (systolic BP [SBP] of 120-139 mm Hg or diastolic BP [DBP] of $80-89 \mathrm{~mm} \mathrm{Hg}$ ) and hypertension as having the SBP of $\geq 140 \mathrm{~mm} \mathrm{Hg}$ or DBP of $\geq 90 \mathrm{~mm} \mathrm{Hg}$. Normal blood pressure is classified using cut-off points of SBP $<120 \mathrm{~mm} \mathrm{Hg}$ and DBP $<80 \mathrm{~mm} \mathrm{Hg}[15,16]$. The report emphasizes that even slightly elevated BP increases cardiovascular risk. Beginning at the SBP/DBP of 115/75 mm Hg, the risk of cardiovascular disease doubles with each increment of 20/10 mm Hg [15]. Whereas numerous studies $[1-9,11]$ have noted the clustering or coexistence of clinically high risk factors and their relation to heart disease and stroke, no studies have examined the cooccurrence of risk factors in South African colliery executives with above-optimal BP levels. The objective of this study was to establish the prevalence of CVD risk factors amongst South African colliery executives presenting normal blood pressure (normotensive), prehypertension and hypertension. Identification of those at risk of developing CVD would be a crucial step in lifestyle modifications as well as creating awareness around chronic disease for all patients with pre-hypertension and hypertension.

\section{MATERIALS AND METHODS}

\section{Participants}

A non-randomized, available population of 143 Caucasian male executives (upper to top management level) from five South African collieries situated in Mpumalanga and Gauteng Provinces aged 26-58 (41.7 7.98 years) was used. The selected CVD risk factors included: total cholesterol (TC), Body Mass Index (BMI), waist-hip-ratio (WHR), body fat percentage, rate pressure product (RPP) and cardiorespiratory fitness. Physical activity and smoking history were also noted. The aforementioned risk factors have been identified and are well established as traditional, independent risk factors for the CVD risk.

All participants were screened using the PAR-Q (Physical Activity Readiness Questionnaire) to ensure the participants safety/readiness prior to the cardiorespiratory fitness test [16]. Those presenting contra-indications for testing were not subjected to the fitness evaluation. Six participants using medication that could affect any of the parameters measured (blood pressure, heart rate, cholesterol, etc.) were excluded from the study. Participants were instructed not to eat, drink or smoke at least $30 \mathrm{~min}$ prior to their blood pressure measurement.

\section{Measurements}

Data was collected and measurements were taken by a registered biokineticist and ethical guidelines for the profession as described by the Health Professions Council of South Africa (HPCSA) were strictly adhered to.

\section{Stature}

Body height was determined using a stadiometer with the participants standing barefoot with the back pressed against the meter and the head kept straight. Height was determined to the nearest $0.5 \mathrm{~cm}[16]$.

Body mass

Body mass was determined using a Tanita solar HS-302 electronic scale and noted to the nearest $0.5 \mathrm{~kg}$. Participants were barefoot only wearing a pair of shorts. The scale was calibrated regularly with standardized weight [16].

\section{Body Mass Index}

Body Mass Index was calculated using the formula: weight $(\mathrm{kg}) /$ height $\left(\mathrm{m}^{2}\right)$ [16]. 
Fat percentage

A Harpenden John Bull skinfold calliper was used to determine skinfold thickness. The 3 (three) skinfold sites measured were the chest/pectoral, abdominal, and thigh [17]. Two measurements were taken, at a minimum, at each site and the average was calculated to determine the subcutaneous fat thickness in millimetres (mm).

Waist-and-hip circumference

A measuring tape was used to determine the executive's waist and hip circumferences to the nearest $0.1 \mathrm{~cm}$ according to the protocol as prescribed by ACSM [16].

\section{Blood pressure}

An ALPK2 aneroid sphygmomanometer was used to determine arterial blood pressure according to the protocol as suggested by the ACSM [16]. The participants were seated for five minutes prior to the measurement and two readings were taken one minute apart.

\section{Total cholesterol}

The Roche Accutrend GC analyzer was used to determine non-fasting total cholesterol. The $\mathrm{CV}$ for precision is $2.9 \%$ and it is widely considered to be appropriate for screening purposes [18]. A capillary blood specimen was obtained from a finger prick for this procedure. The machine was calibrated using the batch-related calibration strip before the assessments. A fasting period prior to the assessment of total cholesterol was not applied due to the evidencebased research indicating that testing in the non-fasting state may not be clinically or significantly different from testing in the fasting state in identifying the patients at risk for a future cardiovascular event [19].

\section{Cardiorespiratory fitness}

Cardiorespiratory fitness was assessed by means of a 3-minute sub-maximal step test. The step test protocol was followed in accordance with the ACSM guidelines [16].
Rate-pressure product

Rate-pressure product was determined by multiplying the resting heart rate with systolic blood pressure [20].

\section{Smoking}

All smokers and non-smokers were categorized into four categories: 1) non-user (not smoked for the past 6 months), 2) 5-9 cigarettes/day, 3) 10-20 cigarettes/day, 4) $>20$ cigarettes/day [21].

Physical activity

The leisure-time physical activity participation of the subjects was categorized into three categories: 1) $\geq 3$ days/week, 2) 1-2 days / week, 3) 1-3 days/month/ sedentary [20].

The American College of Sports Medicine has identified the 'thresholds' above which individuals will be at increased risk for cardiovascular disease. These thresholds were utilized to describe the risk and included the following:

- sedentary lifestyle - not participating in at least $30 \mathrm{~min}$ of moderate intensity exercise at least 3 days of the week for at least 3 months;

- smoking - current smokers or those who quit within the previous 6 months;

- obesity - BMI $\geq 30$;

- blood pressure - systolic blood pressure $\geq 140 \mathrm{~mm} \mathrm{Hg}$ and/or diastolic blood pressure $\geq 90 \mathrm{~mm} \mathrm{Hg}$;

- hypercholesterolemia - total cholesterol (TC) $\geq 5.18 \mathrm{mmol} / \mathrm{L}$;

- family history (MI, coronary revascularization, or sudden death before 55 year of age in the father and before 65 year of age in the mother).

\section{Ethics}

Each participant gave a written consent before joining the study. Ethical guidelines for the profession as described by Health Professions Council of South Africa (HPCSA) 
were strictly adhered to and the Ethics Committee of the University approved the study according to the Helsinki Declaration.

\section{Statistics}

The statistical analysis of the data comprised descriptive summary statistics (N, mean, minimum, maximum and standard deviations). The differences between the means were tested using Anova followed by the post-hock Tukey HSD test (SPSS statistical package). The significance was set at the level of $p<0.05$. The prevalence of those above the threshold for increased risk of cardiovascular disease was expressed in percentages.

\section{RESULTS}

Overall, approximately $28 \%$ of the participants had normal BP (blood pressure) levels, 37\% had pre-hypertension, and 34\% had hypertension. Table 1 indicates that the mean values of all the parameters assessed, except for

Table 1. Characteristics of the participants according to blood pressure categories

\begin{tabular}{|c|c|c|c|c|c|c|}
\hline Risk parameter & Groups & Respondents (n) & $\overline{\mathrm{x}}$ & $\mathrm{SD}$ & Min & Max \\
\hline \multirow[t]{3}{*}{ Age (years) } & normal & 38 & 38.80 & 8.03 & 24.00 & 56.00 \\
\hline & pre-hypertensive & 52 & 42.10 & 7.69 & 26.00 & 60.00 \\
\hline & hypertensive & 47 & 43.60 & 7.72 & 29.00 & 59.00 \\
\hline \multirow{3}{*}{$\begin{array}{l}\text { Cholesterol } \\
(\mathrm{mmol} / \mathrm{L})\end{array}$} & normal & 38 & 4.84 & 0.87 & 3.88 & 7.23 \\
\hline & pre-hypertensive & 52 & 5.36 & 0.98 & 3.40 & 7.43 \\
\hline & hypertensive & 47 & 5.42 & 1.11 & 3.88 & 7.80 \\
\hline \multirow{3}{*}{$\begin{array}{l}\text { Body Mass Index } \\
\quad\left(\mathrm{kg} / \mathrm{m}^{2}\right)\end{array}$} & normal & 38 & 25.97 & 3.78 & 20.50 & 33.80 \\
\hline & pre-hypertensive & 52 & $28.83^{*}$ & 3.29 & 21.90 & 37.40 \\
\hline & hypertensive & 47 & $30.88^{* *}$ & 4.62 & 21.30 & 43.00 \\
\hline \multirow{3}{*}{ Waist-hip-ratio } & normal & 38 & $0.90^{* * *}$ & 0.07 & 0.78 & 1.06 \\
\hline & pre-hypertensive & 52 & 0.94 & 0.07 & 0.69 & 1.07 \\
\hline & hypertensive & 47 & 0.95 & 0.06 & 0.84 & 1.07 \\
\hline \multirow[t]{3}{*}{ Fat $\%$} & normal & 38 & 18.35 & 4.52 & 9.10 & 26.70 \\
\hline & pre-hypertensive & 52 & $20.70^{*}$ & 4.09 & 6.80 & 27.70 \\
\hline & hypertensive & 47 & $22.94 * *$ & 5.17 & 13.50 & 33.90 \\
\hline \multirow[t]{3}{*}{ Weight (kg) } & normal & 38 & 84.10 & 14.44 & 61.00 & 112.00 \\
\hline & pre-hypertensive & 52 & $91.93^{*}$ & 13.56 & 62.00 & 131.00 \\
\hline & hypertensive & 47 & $99.93^{* *}$ & 15.26 & 72.00 & 135.00 \\
\hline \multirow{3}{*}{$\begin{array}{l}\text { Cardiorespiratory } \\
\text { fitness (b/min) }\end{array}$} & normal & 38 & 114.00 & 19.52 & 78.00 & 156.00 \\
\hline & pre-hypertensive & 52 & 111.00 & 18.31 & 72.00 & 150.00 \\
\hline & hypertensive & 47 & 117.00 & 15.17 & 90.00 & 144.00 \\
\hline \multirow{3}{*}{$\begin{array}{l}\text { Rate-pressure } \\
\text { product }\end{array}$} & normal & 38 & 7377.95 & 1252.99 & 4800.00 & 10560.00 \\
\hline & pre-hypertensive & 52 & $8323.63^{*}$ & 1476.57 & 5616.00 & 11700.00 \\
\hline & hypertensive & 47 & $9901.77^{* *}$ & 1707.93 & 6630.00 & 15960.00 \\
\hline
\end{tabular}

$\mathrm{SD}$ - standard deviation.

$* \mathrm{p}<0.05$; pre-hypertensive $>$ normal.

** $\mathrm{p}<0.05$; hypertensive $>$ pre-hypertensive.

*** $\mathrm{p}<0.05$; normal $<$ pre-hypertensive and hypertensive. 
Table 2. Prevalence (\%) of individuals above the threshold for increased risk of cardiovascular disease using selected risk factors

\begin{tabular}{llcc}
\hline \multicolumn{1}{c}{ Variable } & $\begin{array}{c}\text { Normal BP } \\
(\%)\end{array}$ & $\begin{array}{c}\text { Pre-hypertensive } \\
(\%)\end{array}$ & $\begin{array}{c}\text { Hypertensive } \\
(\%)\end{array}$ \\
\hline $\begin{array}{l}\text { Family history (MI, coronary revascularization, or sudden death } \\
\text { before 55 year of age in the father and before 65 year of age }\end{array}$ & 39.5 & 30.8 & 29.8 \\
$\quad$ in the mother) & & & \\
Body Mass Index ( $\geq 30)$ & 21.1 & 46.2 & 57.4 \\
Total cholesterol ( $\geq 5.18$ mmol/L) & 26.3 & 61.5 & 57.4 \\
Smoking (current or quit in last 6 months) & 26.3 & 32.7 & 21.3 \\
$\begin{array}{l}\text { Sedentary lifestyle (< three 30-minute moderate intensity sessions } \\
\text { per week for at least 3 months) }\end{array}$ & 50.0 & 51.9 & 51.1 \\
\hline
\end{tabular}

BP - blood pressure, MI - myocardial infarction.

the cardiorespiratory fitness parameter, increased across the three BP categories. The mean total cholesterol concentrations (TC) of the pre-hypertensive and hypertensive groups fell into the borderline high category ( $\geq 5.18-6.19 \mathrm{mmol} / \mathrm{L}$ ) [16] (Table 1) with $61.5 \%$ and $57.4 \%$, respectively; the TC level above the ACSM threshold for increased risk of CVD [16] (Table 2). No significant differences were noted between the TC of the three groups.

Moreover, Table 1 shows that the mean BMI results of the normal BP and pre-hypertensive groups fell into the overweight category ( $\geq 25-29.9$ ) with the hypertensive group's mean BMI falling into the obese category ( $\geq 30$ ) [16]. Correspondingly, $46.2 \%$ and $57.4 \%$ of the BMI results obtained in the pre-hypertensive and hypertensive groups were above the ACSM threshold for increased risk of CVD [10] (Table 2). Significant differences were noted between the body composition parameters (BMI, WHR, Fat $\%$ and weight) in all three BP groups. Table 1 indicates that in all three groups, the cardiorespiratory fitness level fell into the average category when compared to the ACSM recommended value of $<94 \mathrm{~b} / \mathrm{min}[16$; there were, however, no significant differences between the three groups. The percentage of participants maintaining a sedentary lifestyle and the percentage of the participants above the threshold for increased risk of CVD (performing < three 30 min moderate intensity exercise sessions per week for at least 3 months) were the same in all three BP groups, namely 50\% (normal BP), 51.9\% (pre-hypertensive) and 51\% (hypertensive).

As expected, a significant difference was noted between all three BP groups as for the rate-pressure product statistics; with the mean values in the pre-hypertensive and hypertensive groups measuring above the 'acceptable' value of $<8000$ [16]. The percentage of smokers was lower in the hypertensive group $(21.3 \%)$ as compared to the prehypertensive group $(32.7 \%)$ and to the normal BP group $(26.3 \%)$. Conversely, the percentage of the participants with a family history of myocardial infarction, coronary revascularization, or sudden death before 55 year of age in the case of the father and before 65 year of age in the case of the mother, was higher in the normal blood pressure group $(39.5 \%)$ compared to the pre-hypertensive $(30.8 \%)$ and hypertensive groups $(29.8 \%)$.

\section{DISCUSSION}

Although numerous investigators have reported cardiovascular disease risks associated with elevated blood pressure [1-9,11], few of them have presented the absolute and relative risks of cardiovascular disease according to blood pressure categories in South African colliery executives. In our study, the $34.3 \%$ prevalence 
of hypertension ( $\geq 140 / 90 \mathrm{~mm} \mathrm{Hg}$ ) was slightly lower than the reported $40 \%$ one found among South African corporate executives [22]. It must however be noted that different cut-off points of SBP $\geq 130 \mathrm{mmHg}$ and DBP $85 \mathrm{~mm} \mathrm{Hg}$ were used for that study. We observed that the executives with pre-hypertension and hypertension had a higher prevalence of CVD risk factors, including hypercholesterolemia, obesity, smoking (prehypertension), and a sedentary lifestyle, but they did not have a higher incidence of MI, coronary revascularization, or sudden death in their family history, compared to persons with normal BP levels. The lower prevalence of smokers in the hypertensive (21.3\%) group, compared to the pre-hypertensive group ( $32.7 \%$ ), can be attributed to the fact that "hypertensive" executives noted the impact of smoking in managing blood pressure and, therefore, stopped smoking. This is confirmed with regards to a similar study conducted in the United States of America [11] in which persons with hypertension were less likely to smoke cigarettes compared to those with prehypertension. Furthermore, the percentage of executives with CVD risk factors, with the exception of BMI, was greater among those with pre-hypertension than among those with hypertension.

Out of all the risk factors, body composition (BMI, WHR and fat $\%$ ) exhibited the highest prevalence amongst prehypertensive and hypertensive participants. BMI was furthermore used as an indicator of obesity, defined as a BMI $\geq 25$ [16]. This finding is consistent with other studies [7,23-25]. However, further investigation is warranted regarding the relationship between obesity, prehypertension and hypertension. The limited study on pre-hypertension and hypertension in South African colliery executives precludes a more in-depth examination of this relationship. No significant difference was noted in TC and this is consistent with a study of healthy Korean men where TC was not a significant risk factor for hypertension [23].
This study has several limitations. While risk factors were directly measured, others were self-reported. In addition to this, it is unknown, for example, whether self-reported smoking habits and physical activity status were accurate, true and correct. On the other hand, data on the risk factors examined herein were collected independently, and so these potential biases should be minimal.

\section{CONCLUSION}

In South African colliery executives, body composition/ obesity displayed the highest prevalence of all the risk factors amongst the pre-hypertension and hypertension categories. Early identification and treatment of people with hypertension is therefore vital [7]. Obesity may have independent associations with BP, but they are nevertheless related through physical inactivity and the associations between BP and physical activity reinforce the need to reduce the risk of hypertension through lifestyle changes. Preventing or controlling one risk factor may also affect other risk factor levels. These results, along with others [26-28] suggest the continued need for focused and comprehensive preventive and public health efforts that target hypertension, heart disease, and stroke. The relation between BP and cardiovascular disease risk is graded and continuous [11]. Our observations support the argument that appropriate screening efforts can be initiated in persons with any level of BP in order to create the awareness of the CVD risk and thus potentially avert the development of adverse levels of BP in the first place in order to reduce cardiovascular associated morbidity and mortality.

\section{ACKNOWLEDGEMENTS}

We are grateful to the participants who volunteered for the study and would like to thank Brendon Wocke for proof-reading the manuscript. 


\section{REFERENCES}

1. Bo S, Gambino R, Gentile L, Pagano G, Rosato R, Saracco GM, et al. High-normal blood pressure is associated with a cluster of cardiovascular and metabolic risk factors: a population-based study. J Hypertens 2009;27(1):102-8.

2. Vasan RS, Larson MG, Leip EP, Evans JC, O’Donnell CJ, Kannel WB, et al. Impact of high-normal blood pressure on the risk of cardiovascular disease. $\mathrm{N}$ Engl J Med 2001;345(18):1291-7.

3. Kokubo Y, Kamide K, Okamura T, Watanabe M, Higashiyama A, Kawanishi K, et al. Impact of high-normal blood pressure on the risk of cardiovascular disease in a Japanese urban cohort: the Suita study. Hypertens 2008;52(4):652-9.

4. Wang Y, Wang Q. The prevalence of prehypertension and hypertension among US adults according to the New Joint National committee guidelines. Arch Intern Med 2004;164(19):2126-34.

5. Steyn K, Sliwa K, Hawken S, Commerford P, Onen C, Damasceno A, et al. Risk factors associated with myocardial infarction in Africa: the INTERHEART Africa study. Circulation 2005;112(23):3536-40.

6. O’Donnell MJ, Xavier D, Liu L, Zhang H, Chin SL, RaoMelacini P, et al. Risk factors for ischaemic and intracerebral haemorrhagic stroke in 22 countries (the INTERSTROKE study): A case-control study. Lancet 2010;376(7):112-23.

7. Hendriks ME, Wit FWNM, Roos MTL, Brewster LM, Akande TM, De Beer IH, et al. Hypertension in Sub-Saharan Africa: Cross-Sectional Surveys in Four Rural and Urban Communities. PLoS One 2012;7(3):e32638.

8. Grotto I, Grossman E, Huerta M, Sharabi Y. Prevalence of prehypertension and associated cardiovascular risk profiles among young Israeli adults. Hypertens 2006;48(2):254-9.

9. Ezzati M, Lopez AD, Rodgers A, Vander Hoorn S, Murray CJ. Selected major risk factors and global and regional burden of disease. Lancet 2002;360(11):1347-60.

10. Mbewu AD. Medical Research Council Cardiovascular Disease Research. Medical Research Council; 2005 [cited 2010 Dec 3]. Available from URL: http:/www.mrc. ac.za/publications/cardiovascular/htm.
11. Greenlund KJ, Croft JB, Mensah GA. Prevalence of heart disease and stroke risk factors in persons with prehypertension in the United States, 1999-2000. Arch Intern Med 2004;164(19):113-8.

12. Addo J, Smeeth L, Leon DA. Global Health-Hypertension in Sub-Saharan Africa. Hypertens 2007;50(10):1012-8.

13. Mensah GA. Epidemiology of stroke and high blood pressure in Africa. Heart 2008;94(6):697-705.

14. The Heart and Stroke Foundation of South Africa. Heart disease in South Africa [cited: 2010 Nov 4]. Available from URL: http://www.heartfoundation.co.za/docs/heartmonth/HeartDiseasesSA.pdf.

15. National High Blood Pressure Education Program. The Seventh Report of the Joint National Committee on Prevention, Detection, Evaluation, and Treatment of High Blood Pressure 2004. Bethesda (MD): National Heart, Lung, and Blood Institute (US); 2004.

16. Thompson, WR, Gordon NF, Pescatello LS. ACSM's Guidelines for Exercise Testing and Prescription. 8th ed. Philadelphia: Williams \& Wilkins; 2010.

17. Corbin CB, Lindsey R, Welk G. Concepts of fitness and wellness: A comprehensive lifestyle approach. 3rd ed. Boston: McGraw-Hill Companies; 2000.

18. Roche Accutrend GC analyser. Roche Diagnostics [cited 2011 Jul 18] Available from URL: http://diavant.info/ diavant/CMSFront.html;jsessionid =5CE7CD252CA02DC2 E9EF25AFB73FAAA5?pgid=3,1,4,0.

19. Weiss R, Harder M, Rowe J. The relationship between nonfasting and fasting lipid measurements in patients with or without type 2 diabetes mellitus receiving treatment with 3-hydroxy-3-methylglutaryl-coenzyme A reductase inhibitors. Clin Ther 2003;25(5):1490-7.

20. Durstine JL, Moore GE, Painter PL, Roberts SO. ACSM's Exercise Management for Persons with Chronic Diseases and Disabilities. 3rd ed. Campaign, IL: Human Kinetics; 2003.

21. BioDoc (Biokinetics, Health \& Exercise Epidemiology Consultancy) Health Builder: Medifit Biokinetics Resource Manual. Pretoria: University of Pretoria Sports Institute; 2000. 
22. Ker JA, Rheeder P, Van Tonder R. Frequency of the metabolic syndrome in screened South African corporate executives. Cardiovasc J South Afr 2007;18(1):30-3.

23. Lee JH, Hwang SY, Kim EJ, Kim MJ. Comparison of risk factors between prehypertension and hypertension in Korean male industrial workers. Public Health Nurs 2006;23(4):314-23.

24. Lee KY, Cho BM, Lee SI, Bae DW, Lee HR. Relationships between health practice and hypertension, hypercholesterolemia. J Korean Acad Fam Med 2000;21(5):660-71.

25. Sung KC, Rye SH. Insulin resistance, body mass index, waist circumference are independent risk factors for high blood pressure. Clin Exp Hypertens 2004;26(6):547-56.
26. Hajjar I, Kotchen TA. Trends in prevalence, awareness, treatment, and control of hypertension in the United States, 19882000. JAMA 2003;290(2):199-206.

27. Centers for Disease Control and Prevention. Declining prevalence of no known major risk factors for heart disease and stroke among adults - United States, 1991-2001. MMWR Morb Mortal Wkly Rep 2004;53(1):4-7.

28. Greenland P, Knoll MD, Stamler J, Neaton JD, Dyer AR, Garside DB et al. Major risk factors as antecedents of fatal and nonfatal coronary heart disease events. JAMA 2003;290(7):891-7.

This work is available in Open Access model and licensed under a Creative Commons Attribution-NonCommercial 3.0 Poland License - http://creativecommons.org/ licenses/by-nc/3.0/pl/deed.en. 